\title{
NUEVO REGISTRO FÓSIL DE ATURIA CUBAENSIS (LEA, 1841) [CEPHALOPODA, NAUTILIDAE] PARA EL MIOCENO INFERIOR - MEDIO DE COSTA RICA
}

\section{A NEW FOSSIL RECORD OF ATURIA CUBAENSIS (LEA, 1841) [CEPHALOPODA, NAUTILIDAE] FROM THE LOWER-MIDDLE MIOCENE OF COSTA RICA}

\author{
César A. Laurito ${ }^{1,2 *}$ y Martín J. Mora ${ }^{3}$ \\ ${ }^{1}$ INA, Instituto Nacional de Aprendizaje, Costa Rica \\ ${ }^{2}$ Investigador asociado, Departamento de Historia Natural, Museo Nacional de \\ Costa Rica, San José, Costa Rica \\ ${ }^{3}$ Guía naturalista, Costa Rica \\ *Autor para contacto: cesarlaurito@ice.co.cr
}

(Recibido: 01/07/2017; aceptado: 01/12/2017)

\begin{abstract}
A new specimen of Aturia fossil was collected from sublitoral or shallow marine sedimentary sequences of the Lower- Middle Miocene Roca Carballo Formation at the Central Pacific coast, in the Puntarenas province, Costa Rica. As result of the taxonomic study of the fossil, it is confirmed that this specimen corresponds to Aturia cubaensis (Lea, 1841), based on the shell shape and in major features of its sculpture. This is the second time that this species is recorded for the Miocene of South Central America and some stratigraphic inferences about the first found at the Río Banano Formation are made.

Keywords: Aturia, Nautilidae, Lower-Middle Miocene, Roca Carballo Formation., Río Banano Formation, Costa Rica.

RESUMEN: Un nuevo espécimen de Aturia fósil fue colectado en las secuencias sedimentarias sublitorales o marino someras de la Formación Roca Carballo de edad Mioceno Inferior-Medio, en la costa pacífico central de Costa Rica, en la provincia de Puntarenas. Como resultado del análisis taxonómico del fósil, se confirma el registro de la especie Aturia cubaensis (Lea, 1841), fundamentado en la forma de la concha y de las características de su distintiva escultura. Esta es la segunda vez que se registra dicha especie para el Mioceno del Sur de América Central y por ello se hacen algunas inferencias estratigráficas acerca del primer hallazgo en la Formación Río Banano.

Palabras clave: Aturia, Nautilidae, Mioceno Inferior-Medio, Formación Roca Carballo, Formación Río Banano, Costa Rica.
\end{abstract}

Laurito, C. A. y Mora, M. J. (2018). Nuevo registro fósil de Aturia cubaensis (Lea, 1841) [Cephalopoda, Nautilidae] para el Mioceno Inferior-Medio de Costa Rica. Revista Geológica de América Central, 58, 171-178. doi: 10.15517/rgac.v58i0.32847 


\section{INTRODUCCIÓN}

Miller et al. (1955) describen el primer registro fósil de nautiloideos para Costa Rica, el hallazgo hecho por el señor Alfonso Segura Paguaga, consistía de 2 ejemplares de $40 \mathrm{~mm}$ y $65 \mathrm{~mm}$ de diámetro, procedentes de una arenisca calcárea de color gris claro, aflorante en la quebrada Patincho, al sureste del río Reventazón cerca de Peralta y $5 \mathrm{~km}$ al noreste de Guayabo de Turrialba (cf. Woodring, 1976). Dichos autores determinaron que los especímenes correspondían a la especie Aturia peruviana Olsson y le asignaron una edad Eoceno (?), que luego Woodring (1976) interpretó como Oligoceno (?), cuando describe una nueva especie de Picnodonte, Picnodonte (Crenostrea) segurai, procedente de la localidad de Bonilla, a unos $5 \mathrm{~km}$ de la localidad de Peralta y que él mismo considera son contemporáneos.

Denyer et al. (2003), mencionan el registro del nautiloideo Aturia sp. en las Facies Caldera y más recientemente, Schmidt-Effing y Aguilar, (2008), describen un ejemplar de Aturia sp. recolectado en playas de Doña Ana en el año de 1975, ambas localidades corresponden al Miembro Roca Carballo de la Fm. Punta Carballo y de edad Mioceno Inferior a Medio (sensu Denyer et al., 2003).

El material objeto de estudio del presente trabajo, fue colectado en la base del afloramiento de Roca Carballo, en las coordenadas 9 ${ }^{\circ} 56^{\prime} 27^{\prime \prime}$ N y $84^{\circ} 44^{\prime} 04^{\prime \prime}$ W, en el acantilado homónimo y procede de un banco de lutitas y areniscas rojizas, volcanogénicas, sobreyacidas por areniscas finas a medias, color verdoso con estratificación cruzada de bajo ángulo y abundantes restos de moluscos e ichnofósiles correspondientes al Miembro Roca Carballo (Fig. 1).

\section{PALEONTOLOGÍA}

Clase Cephalopoda (Cuvier, 1795)

Subclase Nautiloidea (Agassiz, 1847)

Orden Nautilida (Agassiz, 1847)
Superfamilia Nautilaceae (Blainville, 1825)

Familia Nautilidae (Blainville, 1825)

Subfamilia Aturinae (Chapman, 1857)

Género Aturia Bronn, 1838

Aturia cubaensis (Lea, 1841)

Sinonimia:

- Nautilus cubaensis Lea (1841; pág.259, lám. 10, fig. 15).

- Aturia peruviana Olsson en Miller et al. (1955; lám. 51, figs. 1-3).

- Aturia cubaensis (Lea, 1841) en Miller y Furnish (1956; pág. 1154, lám. 121, figs. 1-4).

- Aturia cubaensis (Lea, 1841) en Nielsen et al. (2009; pág. 76; figs. 3 y 4).

Para un detallado y exhaustivo análisis de la sinonimia de la presente especie ver los artículos de Jung (1966), Beu (1973), Nielsen et al. (2009).

Material: un fragmocono septado de pequeño tamaño el cual fue colectado por Martín J. Mora durante la realización de la gira de campo del módulo de Geología de Costa Rica del INA y depositado en la colección de fósiles de la Sección de Geología del Departamento de Historia Natural del Museo Nacional de Costa Rica, bajo el código CFM-5273 (Fig. 2).

\section{DESCRIPCIÓN}

Un fragmocono involuto, septado, subdiscoidal con un diámetro de $31 \mathrm{~mm}$ y un ancho de $6 \mathrm{~mm}$ en su extremo más amplio, comprimido por diagénesis, con flancos planos en los que se observan las líneas de sutura y con dificultad la apertura del sifúnculo de menos de $2 \mathrm{~mm}$ de diámetro que tiene una posición dorsal y de contorno lanceolado, pero tal forma es debido a la compresión diagenética de la concha; la cámara corporal no se preservó. Las suturas de lo septos presentan una silla mediana con una suave convexidad y una silla lateral de curvatura amplia cuyos extremos no llegan a unirse en el sector de la costura umbilical, el lóbulo 

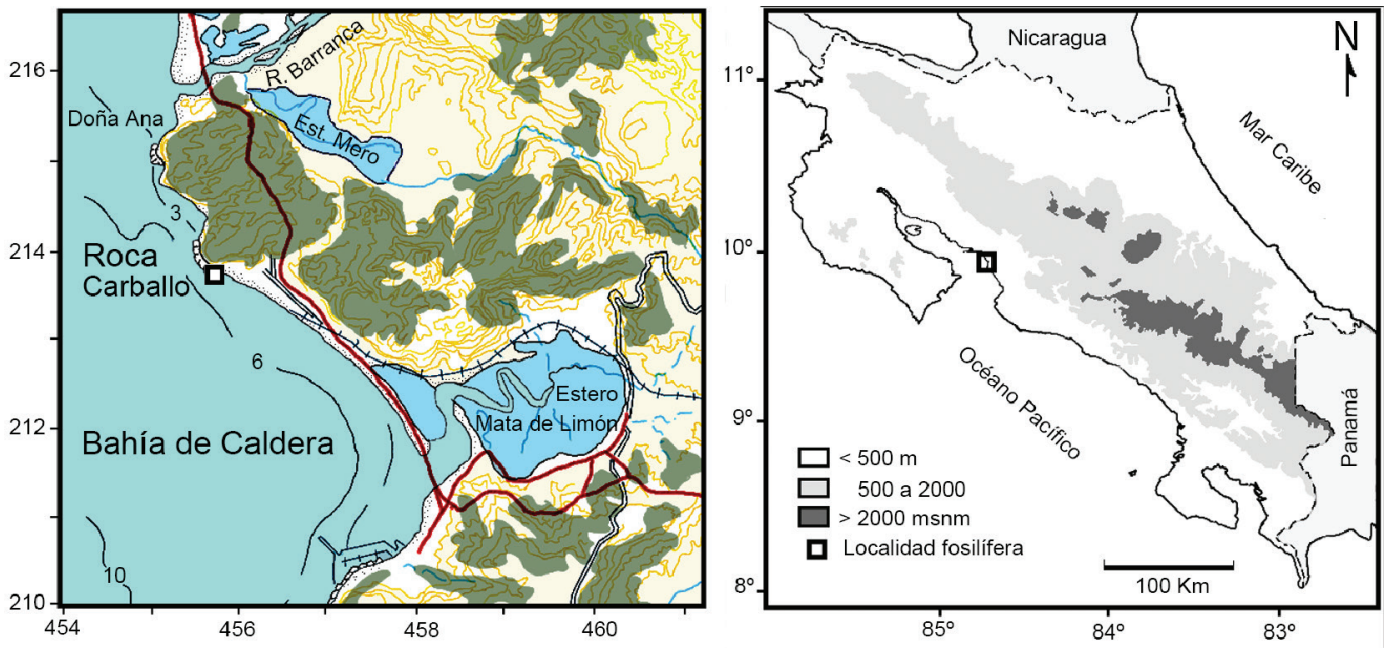

Fig. 1: Mapa de ubicación de la localidad Roca Carballo en la costa pacífica de Costa Rica, América Central.

ventral es lanceolado, con aspecto ligeramente inflado y su extremo posterior agudo, casi llega a tocar el borde lateral de la silla mediana de la correspondiente sutura posterior.

\section{DISTRIBUCIÓN PALEOGEOGRÁFICA}

El género Aturia evolucionó al final del Cretácico y durante el Cenozoico llegó a ser uno de los géneros más diversos de cefalópodos con concha externa, sus fósiles se han hallado en los sedimentos marinos de la mayoría de los continentes y a pesar de su amplia distribución mundial durante el Mioceno, sus hallazgos son más bien raros y escasos (cf. Lukeneder y Harzhauser, 2002).

Como en la mayoría de las especies del género Aturia a lo largo del Cenozoico, la especie Aturia cubaensis (Lea, 1841) presenta una amplia distribución paleogeográfica que en algunos casos se asume es cosmopolita (Jung, 1966; Chirat, 2000; Nielsen et al., 2009).

En el Caribe la especie Aturia cubaensis (Lea, 1841) ha sido registrada en las calizas del Mioceno de La Habana y Mioceno Inferior de Matanzas en Cuba (Lea, 1841; Miller y Downs, 1950; Miller y
Furnish, 1956), en la Fm. Cantaure, península de Paraguaná y en el estado de Lara en Venezuela, y en el Mioceno de Trinidad y Mioceno de la Península de La Guajira en Colombia (Miller y Thompson, 1937; Jung, 1966).

En América Central, la especie Aturia cubaensis (Lea, 1841) se registra en el Mioceno Medio de la Formación Río Banano en el Caribe de Costa Rica y con el presente hallazgo en el Mioceno Inferior-Medio de la Formación Punta Carballo en la costa Pacífica, también en Costa Rica.

En el continente americano, fuera del Caribe y América Central, la especie Aturia cubaensis (Lea, 1841), se distribuye en el Mioceno de la Fm. Chipola en el norte de la Florida (Miller y Downs, 1950) en Norte América y en América del Sur en el Oligoceno tardío de la Fm. San Julián en el Golfo de San Jorge en el sur de la Argentina, y numerosas localidades del Mioceno de la parte central y meridional de Chile (cf. Nielsen et al., 2009). También, se reporta en localidades del Eoceno de Chile, Perú y Venezuela (Miller y Downs, 1950; Miller y Furnish, 1938; Casadío et al., 1999; Jung, 1966; Nielsen et al., 2009).

Fuera del continente Americano, se registra en el Mioceno Inferior, Medio y Superior de 
Nueva Zelanda y Australia (Beu, 1968, 1973), Mioceno Inferior y Medio de Japón (Masayuki et al., 2001) en el Pacífico y en el Mioceno de Italia (Jung, 1966) en Europa; cabe señalar que para el Mioceno inferior de la Cuenca de Viena que forma parte del Parathetys y que además, comprende la cuenca Eslovaca, la región Central y los Cárpatos, recientemente, se describe un depósito excepcional con más de 500 especímenes de Aturia, no asignado a ninguna especie en particular, pero que se considera el yacimiento autóctono más importante hasta ahora registrado (Schlögl et al., 2011).

\section{DISCUSIÓN}

El género Aturia Bronn 1838, se caracteriza por presentar conchas menos globulares y más discoidales que el género Nautilus Linnaeus, 1758 (cf. Miller, 1947) y a diferencia de Nautilus donde el sifúnculo se ubica en la región intermedia, en Aturia se ubica en posición dorsal o muy próxima al dorso, características que se observan en el ejemplar del presente estudio (Fig. 2).

Por otra parte, el género Aturia tiene un amplio rango estratigráfico que abarca desde el Paleoceno hasta el Mioceno (Dzik, 1984; Casadío et al., 1999), aunque se han hallado especímenes de Aturia del Mioceno asociados a foraminíferos del Plioceno temprano (Nielsen et al., 2009) lo que se interpreta como material alóctono redepositado. Además, Jung (1966) concluye que para el Mioceno solo prevalecen 2 especies de Aturia, Aturia aturi (Basterot, 1825) y Aturia cubaensis (Lea, 1841) e incluye en esta última especie el ejemplar de Aturia, inicialmente descrito como Aturia peruviana Olsson por Miller et al. (1955) para la localidad de Quebrada Patincho en el Caribe de Costa Rica.

El patrón de las líneas de sutura en la especie Aturia cubaensis (Lea, 1841) se caracteriza por presentar una silla mediana convexa, sillas laterales amplias y subcirculares, cuyos extremos por lo general no llegan a unirse en la costura umbilical y un lóbulo ventral lanceolado, ligeramente inflado cuyo extremo posterior se aproxima mucho al borde lateral de la silla mediana de la sutura posterior (Fig. 3a). Este patrón se observa claramente en el ejemplar del presente estudio (Fig. 3a), al igual que en el ejemplar descrito por Miller et al. (1955) para el Caribe de Costa Rica (Fig. 3b), así como en ejemplares de otras latitudes (Fig. 3c) y permite diferenciarlo del patrón de la línea de sutura que presenta la otra especie del Mioceno, Aturia aturi (Basterot, 1825), caracterizado por
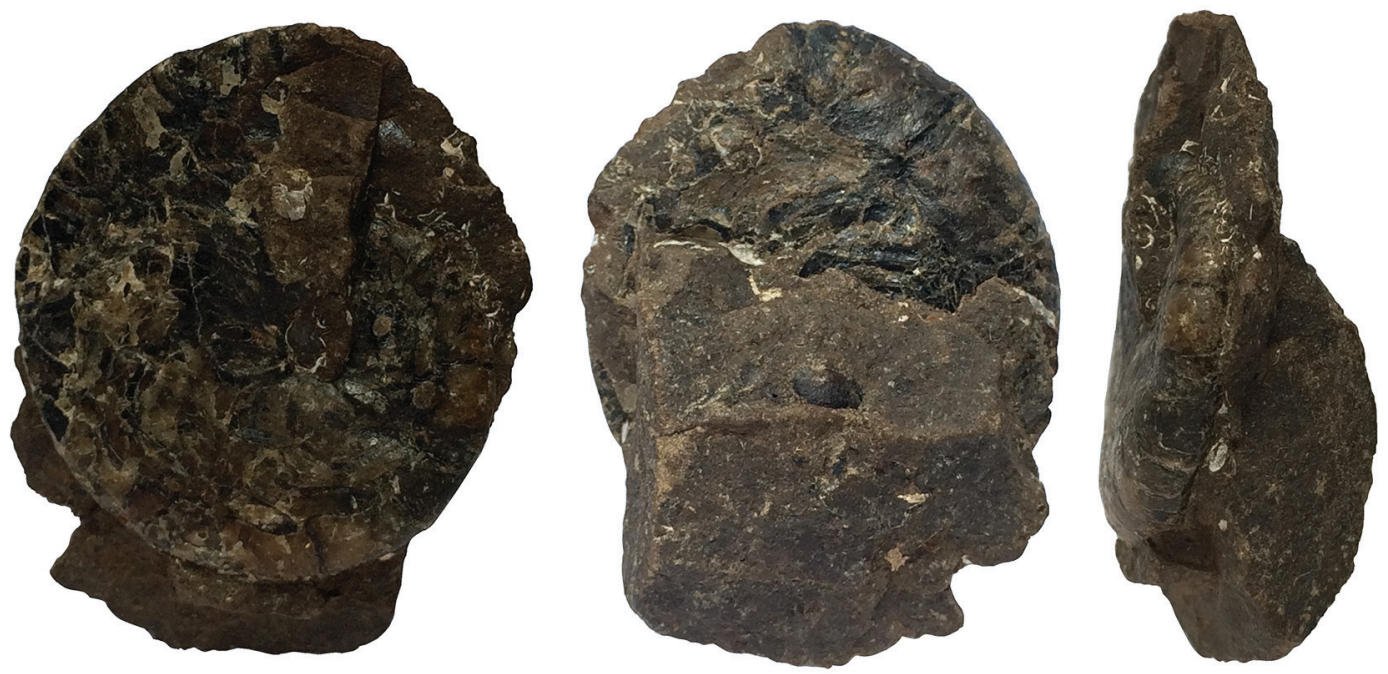

Fig. 2: ejemplar CFM-5273, fragmocono de Aturia cubaensis (Lea, 1841) en a. y b. vistas laterales y c. vista mediana, Fm. Punta Carballo, Mioceno Inferior - Medio. Escala visual $10 \mathrm{~mm}$. 

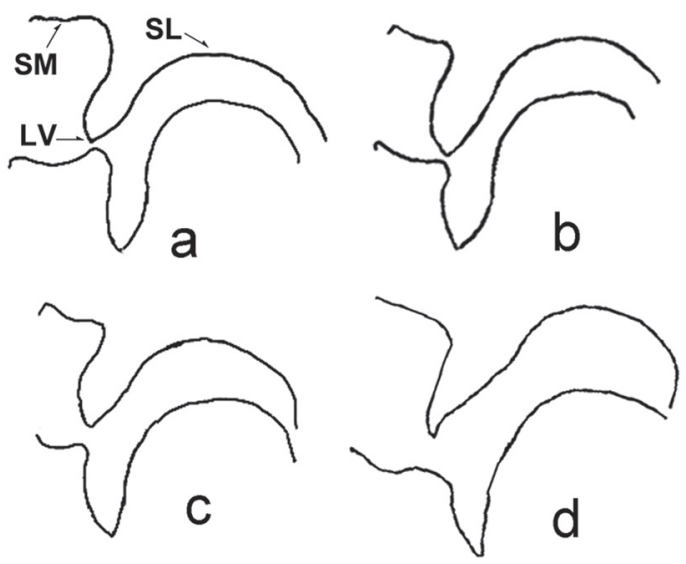

Fig. 3: Pares consecutivos de líneas de sutura septal de Aturia cubaensis (Lea, 1841) de las localidades de a. Roca Carballo, Fm. Roca Carballo y b. Quebrada Patincho, Fm. Río Banano ilustrado por Miller et al., 1955 Miller et al., 1955, ambas de Costa Rica; c. ejemplar de Aturia cubaensis (Lea, 1841) procedente de la localidad Bahía Inútil, Tierra del Fuego, Chile, ilustrado por Nielsen et al., 2009 y d. ejemplar de Aturia aturi (Basterot, 1825) de la localidad de Obermarkersdorf, Austria, ilustrado por Lukeneder y Harzhauser (2002). Simbología: SM silla mediana, SL silla lateral y LV lóbulo ventral.

lóbulos ventrales triangulares con el extremo posterior agudo, septos separados por amplios espacios entre sí y las sillas laterales presentan extremos convergentes que se unen en la costura umbilical (Fig. 3d).

Ambas especies presentan una amplia distribución planetaria (Jung, 1966), tanto tropical como subtropical que se asume obedece a transporte post morten por deriva oceánica. La concha del género Aturia se caracteriza por tener el sifúnculo en posición dorsal y cuellos septales muy largos (Ward, 1980; Dzik, 1984) lo que permite una eficiente capacidad de desacople del líquido de las cámaras (Ward, 1980) y por ello tiene el mayor potencial de flotabilidad y de deriva post morten en comparación con la concha de los nautiloideos (Chirat, 2000); en el caso del género Nautilus la flotabilidad post morten puede ser positiva o negativa (Mapes et al., 2010), si es positiva, se sabe que la concha de Nautilus puede derivar por días o meses (House, 1987), distancias de varias decenas, cientos e incluso superar los 1000 kilómetros fuera del área en que habitan antes de depositarse (Saunders y Spinosa, 1979). Ello, en principio, explicaría la amplia distribución paleogeográfica de la especie Aturia cubaensis (Lea, 1841); de hecho, Chirat (2000) cree que América Central y las regiones adyacentes, fue la región donde Aturia cubaensis (Lea, 1841) habitó y que las conchas derivaron fácilmente desde esta zona, atravesando el Atlántico hasta el Mediterráneo y el Pacífico hasta Japón y Pacífico Sur, alcanzado el sur de América del Sur. Sin embargo, Nielsen et al., 2009, sugieren que las los hallazgos de Aturia cubaensis (Lea, 1841) en yacimientos del Eoceno-Oligoceno de Tierra del Fuego y Mioceno Tardío de las costas centrales y meridionales de Chile, obedece no a la deriva por corrientes oceánicas, sino a poblaciones autóctonas que se vieron favorecidas por mares más cálidos que imperaron, por ejemplo, en el Mioceno, ello se constata con la presencia de variados géneros de gasterópodos netamente tropicales asociados al registro de Aturia en el sur de América del Sur (Nielsen et al., 2006).

Schlögl et al. (2011), determinan con base en análisis isotópicos aplicados a un acúmulo extraordinario de restos de Aturia del Mioceno Inferior, donde se encontraron individuos en diferentes estadios ontogenéticos, junto a restos de mandíbulas superiores en inferiores, lo que interpretaron como evidencia inequívoca de un yacimiento tafonómicamente autóctono, que el género Aturia habitaba aguas relativamente profundas (240 y $330 \mathrm{~m}$ ) y bajas en oxígeno, a temperaturas entre los 13,3 a $17,9^{\circ} \mathrm{C}$ y temperatura de las aguas superficiales entre los 20 y $22^{\circ} \mathrm{C}$ y que al igual que Nautilus, Aturia compartía hábitos nectobentónicos con migraciones verticales diarias.

Por otra parte, al analizar las secuencias sedimentarias aflorantes en la quebrada Patincho, afluente del río Reventazón, en la localidad de Bajo 52 Millas en la provincia de Limón, donde Alfonso Segura Paguaga recolectó los 2 ejemplares de Aturia cubaensis (Lea, 1841), es claro que los sedimentos allí aflorantes son marino someros y forman parte de la Formación Río Banano de edad Mioceno Inferior a Medio (cf. Fernández, 1987). De igual manera el ejemplar de del presente estudio, procede de secuencias marino someras, sublitorales o nerítico internas, de la Formación Punta Carballo de similar edad, Mioceno Inferior a Medio (cf. Denyer et al., 2003). 


\section{CONCLUSIONES}

Se registra por segunda vez en el sur de América Central y para el Mioceno Inferior Medio de Costa Rica, la especie Aturia cubaensis (Lea, 1841). La primera se corresponde con el hallazgo de 2 ejemplares colectados por el $\mathrm{Sr}$. Alfonso Segura Paguaga que fue dado a conocer por Miller et al. (1955) y que luego fue determinado taxonómicamente por Jung (1966), no queda duda, que dichos hallazgos, procedentes de sedimentos aflorantes en la quebrada Patincho en la provincia de Limón, pertenecen a la Formación Río Banano y su edad corresponde al Mioceno Inferior a Medio de acuerdo con los datos sedimentológicos y estratigráficos obtenidos para dicha localidad por Fernández (1987).

Aunque el género Aturia ha sido registrado en varias ocasiones para la Formación Punta Carballo, es la primera vez que se logra una determinación taxonómica a nivel de especie. Es claro que el hallazgo de Aturia cubaensis (Lea, 1841) en formaciones marino someras del Mioceno Inferior a Medio, tanto del Pacífico Central como del Caribe de Costa Rica, sugieren una conexión paleobiogeográfica y paleoceanográfica entre las formaciones Punta Carballo y Río Banano; así como con otras localidades del Caribe e incluso del Pacífico meridional de América del Sur.

El hecho de que los restos de Aturia cubaensis (Lea, 1841) se presenten en sedimentos nerítico internos, probablemente sublitorales, obedece a la capacidad de flotación positiva post morten de las conchas de Aturia, eso mismo ha sido observado en conchas con flotación positiva de Nautilus (Seuss, et al., 2015), pero en Aturia ello se incrementa por la forma más compleja de los cuellos septales telescópicamente alargados lo que evita el ingreso de agua, una vez muerto el organismo y provoca que la concha suba a la superficie, sea efectivamente arrastrada por las corrientes (Nielsen et al., 2009) y depositada en ambientes diferentes a los que habitaba.

\section{REFERENCIAS BIBLIOGRÁFICAS}

Beu, A. G. (1968). A specimen of the nautiloid Aturia from the Kapitean Stage (uppermost Miocene) of New Zealand. New Zealand Journal of Geology and Geophysics, 11 (1), 161-165.

Beu, A. G. (1973). Nautiloids of the Genus Aturia from the Uppermost Miocene of Australia and New Zealand. 6, 297-308.

Casadío, S., Rodríguez, M. F., Reichler, V. A., Camacho, H. H. (1999). Tertiary Nautiloids from Patagonia, Southern Argentina. Ameghiniana, 36, 189-202.

Chirat, R. (2000). The so-called 'Cosmopolitan palaeobiogeographic distribution' of Tertiary Nautilidae of the genus Aturia Bronn 1938: the result of post-mortem transport by oceanic palaeocurrents. Palaeogeography, Palaeoclimatlogy, Palaeoecology, 157, 59-77.

Denyer, P., Aguilar, T. y Alvarado, G. (2003). Geología y estratigrafía de la Hoja Barranca, Costa Rica. Revista Geológica de América Central, 29, 105-125.

Dzik, J. (1984). Phylogeny of the Nautiloidea. Palaentologia Polonica, 45, 1-219.

Fernández, J.A. (1987). Geología de la hoja topográfica Tucurrique (1:50 000, I.G.N.C.R., \# 3445 I) (Tesis de licenciatura inédita). Universidad de Costa Rica, San José, Costa Rica.

House, M. R. (1987). Geographic distribution of Nautilius Shell. En W. B. Saunders y N. H. Landman (eds), Nautilus: the biology and paleobiology of a living fossil (pp. 53-54). New York: Plenum Press. 
Jung, P. (1966). Zwei Miocaene Arten von Aturia (Nautilaceae). Eclogae Geologicae Helvetiae, 59(1), 485-493.

Lea, I. (1841). Notice of the Oolitic Formation in America, with descriptions of some of its organic remains. Transactions of the American Philosophical Society, 7, 251-260.

Lukeneder, A. y Harzhauser, M. (2002) Shell acumulations of the Nautilidae Aturia (Aturia) aturi (Bast.) in the Lower Miocene Paratethys (Lower Austria). Abhandlungen der Geologischen Bundesanstalt, 57, 459466.

Mapes, R. H., Landman, N. H., Cochran, K., Goiran, C., Richer De Forges, B. y Renfro, A. (2010). Early taphonomy and significance of naturally submerged Nautilus shells from the New Caledonia region. Palaios, 25 (9-10), 597-610.

Masayuki, E., Masamichi, S. y Yukihiro, T. (2001). Occurrence of Aturia (Cephalopoda) from the Miocene Moniwa Formation of Sendai, Northeast Honshu, Japan and its paleoenvironmental significance. Earth Science, 55, 183-186.

Miller, A. K. (1947). Tertiary Nautiloids of the Americas. Geological Society of America Memoir, 23, 1-234.

Miller, A. K. y Furnish, W. M. (1938) Aturias from the Tertiary of Mexico. Journal of Paleontology, 12(2), 149-155.

Miller, A. K. y Downs, H. R. (1950). Tertiary nautiloids of the Americas: supplement. Journal of Paleontology, 24(1), 1-18.

Miller, A. K. y Furnish, W. M. (1956) An Aturia from Eastern of Cuba. Journal of Paleontology, 30(5), 1154.
Miller, A. K., Furnish, W. M. y Segura Paguaga, A. (1955). Aturias from Costa Rica. Journal of Paleontology, 29(3), 465-466.

Miller, A. K. y Thompson, M. L. (1937) Beiträge zur Kenntnis tropisch-amerikanischer Tertiärmollusken. Teil VI, Some tertiary Nautiloids from Venezuela and Trinidad. Eclogae Geologicae Helvetiae, 30, 59-72.

Nielsen, S. N., Bandel, K. y Kröger, B. (2006). Aturia cubaensis (Lea, 1841) (Cephalopoda, Nautiloidea) in the Miocene of Chile: Paleobiography, Taphonomy and Mode of life. Ponencia presentada en el XI Congreso Geológico Chileno, Antofagasta, II Región, Chile. Actas, 2, 89-92.

Nielsen, S. N., Bandel, K. y Kröger, B. (2009), Palaeobiogeographical provenance, taphonomy, and mode of life of Aturia cubaensis (Cephalopoda, Nautiloidea) from Cainozoic deposits of Chile. Geobios, 42, 73-88.

Schlögl, J., Chirat, R., Valter, V., Joachimski, M., Hudácková, N. y Quillévéré, F. (2011), Aturia from the Miocene Paratethys: An exceptional window on nautilid habitat and lifestyle. Palaeogeography, Palaeoclimatlogy, Palaeoecology, 308, 330-338.

Saunders, W. B. y Spinosa, C. (1979). Nautilus movement and distribution in Palau, Western Caroline Islands. Science, 204, 1199-1201.

Schmidt-Effing, R. y Aguilar, T. (2008). Dos fósiles notables de la Formación Punta Carballo. Revista Geológica de América Central, 39, 117-120.

Seuss, B., Hembree, D. I., Wisshak, M., Mapes, R. H. y Landman, N. H. (2015). Bioerosion in shells of modern Nautilus Taphonomy of deep-marine versus back- 
shore collected Nautilus macromphalus conchs (New Caledonia). Palaios, 30(7), 503-513.

Ward, P. (1980). Comparative shell shape distribution in Jurassic-Cretaceous Ammonites and
Jurassic-Tertiary Nautilids. Paleobiology, 16(1), 32-43.

Woodring, W. P. (1976). A massive Oligocene (?) Pycnodonteine oyster from Costa Rica. Journal of Paleontology, 50(5), 851-857. 